\title{
Transcanal Endoscopic Antrostomy Hole : Its Utility in Restoring Ventilation Pathway to Epitympanum and Mastoid Antrum During Tympanoplasty
}

\author{
Sheikh Shawkat Kamal \\ Department of ENT \\ Surgiscope Hospital Ltd. \\ 445/466 Katalgoni, \\ Chittagong-4203, Bangladesh.
}

Correspondence to:

\author{
Dr. Sheikh Shawkat Kamal \\ Consultant Surgeon \\ Surgiscope Hospital Ltd. \\ 445/466 Katalgoni \\ Chittagong-4203, Bangladesh \\ Mobile: +880-1711406943 \\ Email: drioyent@yahoo.com
}

\begin{abstract}
Aim: To evaluate the role of newly created transcanal endoscopic antrostomy hole as a passage in restoring the aeration of epitympanum and mastoid antrum. Study design: Retrospective case series study. Study duration: From January 2013 to January 2014. Study place: Private tertiary care hospital. Patients: 27 ears of 23 adult patients (age ranging from 15years to 54years) underwent transcanal endoscopic tympanoplasty for their chronic middle ear diseases in presence of inflamed middle ear mucosa along with radiological shadows of stagnant fluid in their epitympanum and mastoid antrum. Structured three months follow-up was ensured in every case. Intervention: A hole was created at a selected site of posterior meatal wall purely through transcanal endoscopic approach that established direct communication between mastoid antrum and external auditory canal. Thereafter this newly created passage was used for three purposes- assessment of the condition of mastoid antrum, performing the water test for checking epitympanic patency and in few cases placement of temporary tube for postoperative ventilation and drainage of middle ear. Main out come measures: The feasibility, performance and management of transcanal endoscopic antrostomy hole as passage for reestablishing the aeration of epitympanum and mastoid antrum. Results: The chosen site for antrostomy hole was found effective and safe in providing convenient entrance into the mastoid antrum in every case in this study with out facing technical complexity and failure. Postoperative healing of skin over antrostomy hole was found complete in all ears without any inward growth of skin in to mastoid antrum. Available post operative CT scan imaging of temporal bones showed improved aeration in their epitympanum and mastoid antrum. Conclusions: The role of transcanal antrostomy hole has been proved worthy in restoring ventilation pathway to epitympanum and mastoid antrum during tympanoplasty. This antrostomy hole has the potentiality to be considered in future for placement of long term mastoid ventilation tube in order to treat persistent atelectatic middle ear.
\end{abstract}

Key words: Endoscopic; transcanal antrostomy hole.

\section{INTRODUCTION}

Securing the ventilation of different compartments of middle ear cleft during tympanoplasty has enormous impact on its surgical outcome. Failure to maintain ventilation causes complications such as retraction of graft even grafts failure. Retraction of graft in the long runs may cause erosion or fixation of ossicular chain thus ultimately jeopardizes the initial hearing gain.

Ventilation of Middle ear cleft usually depends on the function of eustachian tube, patency of tympanic isthmus, posterior epitympanum, volume of mastoid antrum and the condition of mucosa of middle ear cleft. Radiological image illustrating hypocellular or scleorsed mastoid along with stagnant fluid shadow in epitympanum and mastoid antrum indicates poor ventilation of those compartments. 
Thick inflamed mucosa, mucosal adhesions around tympanic isthmus or ossicles, cholesteatoma, granulation tissue polyps are the common reasons causing blockage of the ventilation pathway to epitympanum and mastoid antrum. To restore this ventilation pathway two approaches have been usually practicing. One is antero-posterior approach involving extended atticotomy, removal of blockage and reconstruction of attic wall. Another is posterior-anterior approach involving cortical mastoidectomy and water test to evaluate epitympanic patency along with removal of blockage.

This study described a novel procedure that was used to secure the ventilation of epitympanum and mastoid antrum purely through transcanal endoscopic guidance. First a passage was created in between external auditory canal and mastoid antrum through transcanal endoscopic antrostomy. This new passage was then used for assessing the condition of mastoid antrum and performing the water test for checking epitympanic patency. Blockage of ventilation pathway was removed accordingly under endoscopic guidance through transcanal route. In few cases this newly created passage was also used for placing of temporary tube for immediate postoperative ventilation and drainage of middle ear cleft.

\section{PATIENTS AND METHODS}

This study evaluated the medical records of selected 27 ears of 23 adult patients (age ranging from 15years to 54years) underwent transcanal endoscopic tympanoplasty in presence of congested edematous middle ear mucosa along with clinical and radiological evidences of insufficient ventilation of epitympanum and mastoid antrum in a tertiary care private hospital in Chittagong, Bangladesh from January 2013 to January 2014. Mandatory follow up was three months from operation. All patients were informed in details about this new procedure and its probable consequents. Ethical committee of the Surgiscope Hospital approved the study to be performed in its campus.

Clinical and radiological findings are summarized in table 1. Out of 27 ears, 22 ears had central perforation with mucosal adhesion around tympanic isthmus and among these 22 ears, 17 ears had localized inflamed mucosa around tympanic isthmus while rest 10 ears had diffuse inflamed mucosa with presence of granulation tissue polyps in 3 ears. Among the rest 5 of 27 ears, 3 ears had retracted posterior tympanic membrane with atelectasis and 2 ears had diffuse retracted tympanic membrane with limited attic cholesteatoma. Complete tensor fold was observed in 11 ears with the help of $30^{\circ}$ degree $2.7 \mathrm{~mm}$ oto-endoscope.
Table 1: Clinical and radiological findings of the ears.

$\begin{array}{lc}\begin{array}{l}\text { Findings: } \\ \text { Clinical findings of mesotympanum and epitympanum }\end{array} & \begin{array}{c}\text { Number of ears } \\ (\mathrm{N}=27)\end{array} \\ \text { Central perforation with mucosal adhesion at tympanic isthmus. } & 22 \\ \text { Localized inflamed mucosa around tympanic isthmus. } & 17 \\ \text { Diffuse inflamed mucosa of mesotympanum without polyp. } & 7 \\ \text { Granulation tissue polyps in mesotympanum with polyp. } & 3 \\ \text { Retracted tympanic membrane with atelectasis. } & 3 \\ \text { Retracted tympanic membrane with limited attic cholesteatoma. } & 2 \\ \text { Complete tensor fold. } & 11 \\ \text { Erosion of manubrium of malleus. } & 10 \\ \text { Erosion of lenticular process of incus. } & 5 \\ \text { Clinical findings of mastoid antrum:- } & 17 \\ \text { Healthy appearance of mucosa of mastoid antrum. } & 10 \\ \text { Slightly inflamed mucosa of mastoid antrum. } & \\ \text { Radiological finding of temporal bones:- } & 27 \\ \text { Partial to complete sclerosis of mastoid air cells. } & 11 \\ \text { Shadow of stagnant fluid in mastoid antrum } & 11 \\ \text { Shadow of stagnant fluid in whole epitympanum } & 16 \\ \text { Shadow of stagnant fluid in only in posterior epitympanum } & \\ & \end{array}$

In pre operative CT scan of temporal bones, all cases had partial to complete sclerosis of mastoid air cells. In 27 temporal bones, posterior epitympanic compartment and mastoid antrum were seen completely occupied by soft tissue shadow suggestive of stagnant inflammatory fluid [figure 1].

In 11 cases, stagnant fluid shadow was seen present in anterior compartment of epitympanum in addition to their posterior epitympanic compartment and mastoid antrum.

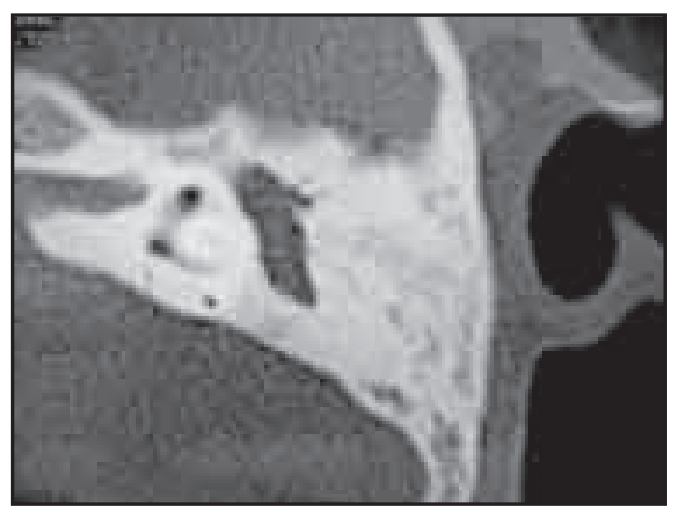

Figure 1: CT scan of temporal bone showed shadow suggestive of stagnant fluid in mastoid antrum and superior epitympanum.

All surgical procedures were performed by a single surgeon (the author) through transcanal route purely under endoscopic guidance using local anesthesia. Most of the soft tissue dissection was done with $0^{0}$ degree $4 \mathrm{~mm}$ endoscope. $0^{0}$ degree and $30^{\circ}$ degree $2.7 \mathrm{~mm}$ oto-endoscope were only used during bony dissection and soft tissue dissection of anterior compartment. 
Transcanal endoscopic antrostomy hol

Table 2: Procedures applied for restoring ventilation to epitympanum and mastoid antrum.

\begin{tabular}{lc} 
Procedures & $\begin{array}{c}\text { Number of ears } \\
(\mathrm{N}=27)\end{array}$ \\
Creation of endoscopic transcanal antrostomy hole. & 27 \\
Release of adhesions around tympanic isthmus and ossicles. & 23 \\
Removal of complete tensor fold. & 9 \\
Removal of incus only. & 2 \\
Removal of cholesteatoma matrix along with incus and head of malleus. & 2 \\
Insertion of ventilation tube in the antrostomy hole. & 10 \\
\hline
\end{tabular}

A wide tympanomeatal flap involving the whole non hairy skin of meatus was elevated. Integrity of ossicular chain and tympanic pathologies were noted. Mucosal adhesions around tympanic isthmus were released accordingly [figure 2]. Retracted tympanic membrane over ossicles was elevated and in some cases localized tymapanectomy was performed.

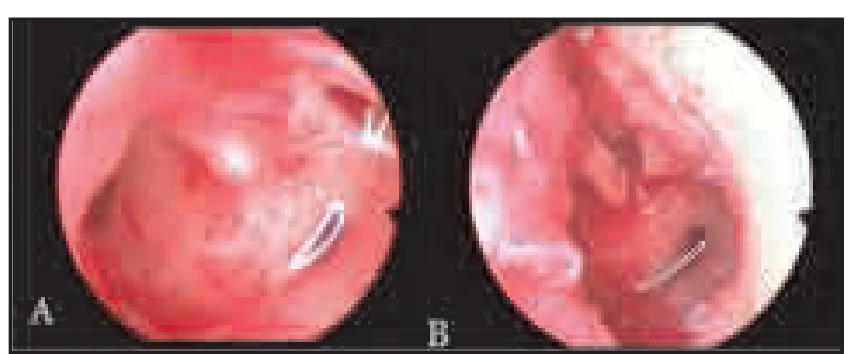

Figure 2: Mucosal adhesions surrounding tympanic isthmus with inflammation was shown in picture (A). After releasing of this mucosal adhesion, restoration of the ventilation pathway through the tympanic isthmus was shown in picture $(\mathrm{B})$.

An antrostomy hole was then gradually created by cutting drill bar of $1.8 \mathrm{~mm}$ diameter in posterior bony meatal wall. The chosen site for antrostomy hole was a point near the hair line of skin of external auditory canal where roof and posterior wall of external auditory canal meet [figure 3]. The site of hole was approximately 4-5 $\mathrm{mm}$ above and posterior to the bony tympanic annulus. This newly created passage connected the external auditory canal with mastoid antrum. The stagnant inflammatory fluid in mastoid antrum was then sucked out. The condition of antrum mucosa was observed with 2.7 diameter wide $0^{0}$ oto-endoscope. In some cases the hole was enlarged in diameter to get better view of mastoid antrum mucosa [figure 4].

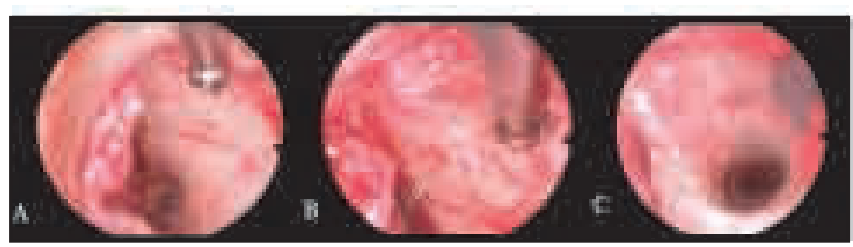

Figure 3:Tip of the bar indicated the site of dissection for antrostomy hole [Shawkat's point] (A). Continuation of bony dissection was shown in subsequent pictures (B) and (C).

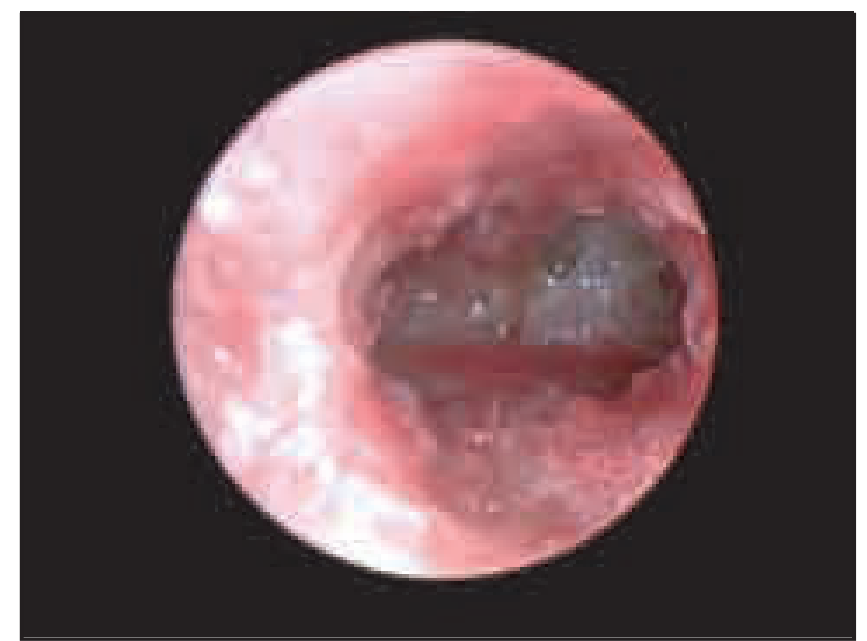

Figure 4 : Healthy mucosa of mastoid antrum was noted through antrostomy hole.

Epitympanic patency was checked through water test. Water test involved gentle injection of few milliliter of saline water through newly created passage and suction of water through mesotympanum. During suction of water through mesotympanum, the inward movement of water level in new passage was evaluated [figure 5].

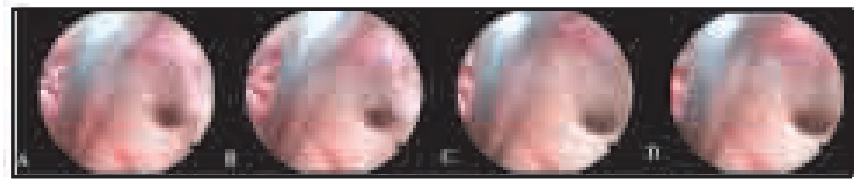

Figure 5: Successive pictures (A), (B), (C) and (D) showed the flow of water through antrostomy hole (please note the changing position of the reflected light of the water) during suction of water in mesotympanum in water test.

In some cases removal of incus was required to reestablish the patency of blocked epitympanum. Ears having ill ventilation in anterior compartment of epitympanum were approached inferiorly with $30^{\circ}$ degree oto-endoscope and involved removal of membranous tensor fold. In cases with limited epitympanic cholesteatoma where anterior compartment of epitympanum was approached both inferiorly and laterally involving limited atticotomy and sometimes removal of head of malleus. Total excision of attic cholesteatoma was ensured.

Temporalis fascia was harvested as graft for reconstruction of perforated tympanic membrane as well as to seal the antrostomy hole. Tragal composite cartilage graft was also used to reinforce the retracted tympanic membrane. Ossiculoplasy involved incus interposition and placement of partial ossicular replacement prosthesis.

For postoperative drainage and ventilation, insertion of a ventilation tube into antrostomy hole was carried out only in cases where mucosa of middle ear cleft was heavily inflamed. The length of antrostomy hole was measured. Thereafter a Richardson's T tube was sized accordingly and placed into the antrostomy hole keeping the flange end in external auditory canal [figure 6]. 


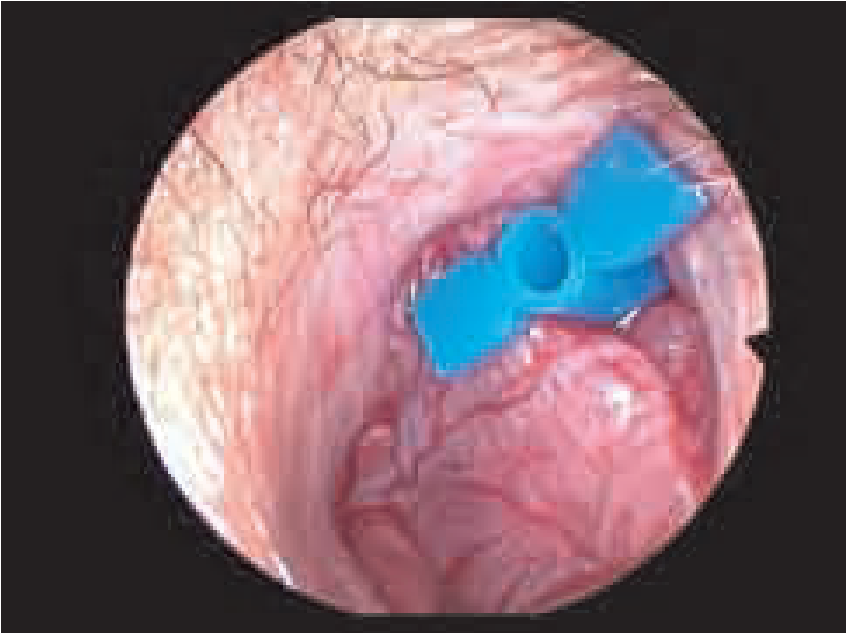

Figure 6: Picture showed the inserted ventilation $\mathrm{T}$ tube in the antrostomy hole.

All patients were scheduled to attend post operative visit at 5 days interval for first 15 days then at 7 days interval for next 14 days then at 1 month interval for next 2 months. During post operative visit the patency of tube was checked. In presence of blockage, the tube was removed and cleaned with $6 \%$ hydrogen per oxide. After reestablishing the patency, the tube was then reinserted though antrostomy hole. Temporary ventilation tube was removed on 15 days after tympanoplasty. After removal of tube the skin margin of antrostomy hole was freshened under local anesthesia and a piece of gel foam was placed into that antrostomy hole.

In subsequent follow up the healing of antrostomy wound and any occurrence of graft retraction or failure were observed.

Postoperative CT scan of temporal bone was advised at the end of 3 months from operation.

\section{RESULTS}

The chosen site for antrostomy hole was found providing appropriate and convenient entrance into the mastoid antrum in every case in this study with out any difficulty and failure. The usual diameter of the antrostomy hole was ranging from $2.5 \mathrm{~mm}$ to $3 \mathrm{~mm}$.

At final stage of dissection while it was touching the mucoperiosteal layer of mastoid antrum, bleeding was noticed. In presence of healthy mastoid mucosa this bleeding was usually found negligible but it was a bit more in presence of inflamed edematous mastoid mucosa. Touching the mucoperiosteum of antrostomy hole sometime produced slight pain.Condition of mucosa of mastoid antrum appeared healthy in 17 ears. In rest 10 ears had slightly inflamed mucosa. In all cases inflammatory fluid was appeared as mucous type.
After releasing mucosal adhesion and retracted tympanic membrane around tympanic annulus and ossicles, epitympanic patency was found secured in 23 ears. To ensure epitympanic patency in rest 4 ears, 2 ears needed removal of incus and 2 ears with limited epitympanic cholesteatoma needed removal of incus, head of malleus along with cholesteatoma matrix. Table 2 summarized the procedures mentioned above needed for restoration of ventilation pathway. Among 11 ears having ill ventilation in anterior compartment of epitympanum, in 9 ears the anterior epitympanic compartment were approached inferiorly with $30^{\circ}$ oto-endoscope and involved removal of membranous tensor fold. In rest 2 ears with limited epitympanic cholesteatoma where aeration to whole epitympanum was ensured by removal of incus, head of malleus along with cholesteatoma matrix.

Ventilation tube was inserted in 10 ears with diffused inflamed mesotympanic mucosa. The average length of $\mathrm{T}$ tube was $7 \mathrm{~mm}$ which included $3 \mathrm{~mm}$ bony length of antrostomy passage plus $2 \mathrm{~mm}$ skin thickness of external auditory canal plus $2 \mathrm{~mm}$ protruding length into mastoid antrum.

Mandatory three months follow-up after operation was ensured in every case. Active discharge through ventilation tube was observed in all 10 ears with tube at $5^{\text {th }}$ postoperative day. Active tubal discharge was seen reducing in following days and at $10^{\text {th }}$ postoperative day only 3 ears had active tubal discharge. At the end of $15^{\text {th }}$ postoperative day none ears had active tubal discharge. Patency of non discharging tubes were checked at $10^{\text {th }}$ postoperative day and in 3 out of 10 ears tubes were found blocked with dry mucoid plug which were cleaned and reinserted. Removal and reinsertion of tubes was performed with out any difficulty. Discomfort felt by patient during manipulation of tube produced was slight and tolerable in all cases. At the end of $15^{\text {th }}$ postoperative day before discarding, all tube was found patent.

Healing of skin over antrostomy hole was found almost complete with slight dimple in those ears with larger antrostomy hole at the end of 1 month following operation. At the end of 2 months following operation the dimple of skin disappeared and skin was appeared normal. Inward growth of skin in to mastoid antrum from the site of antrostomy hole was not seen in any case. All graft was seen well taken. There was no retraction of newly formed tympanic membrane at the end of $3^{\text {rd }}$ month from operation.

Post operative CT scan imaging of temporal bones was carried out after 3 months from operation only in 9 ears due to poor economic background of most of the patients of this study. Completely improved restoration of aeration of middle ear cleft was observed in 7 ears comprising 3ears with history of ventilation tube insertion and 4 ears without history of ventilation tube insertion. In rest 2 ears with history of ventilation tube insertion showed partially improved restoration of aeration. 


\section{DISCUSSION}

Well aeration of middle ear cleft is necessary to maintain its functional and anatomical harmony. Poor ventilation invites retraction of tympanic membrane, chronic otitis media with effusion even cholesteatoma formation along with different levels of hearing impairment. Evidences from different studies showed that to keep both functional and anatomical harmony of the middle ear, well ventilation not only to mesotympanum but also to epitympanum and mastoid was important. The observation of the study conducted by Takahashi et al concluded that the well re-aeration of mastoid after canal wall up tympanoplasty would prevent the postoperative attic retraction ${ }^{1}$. Poor ventilation to mastoid accelerated the progress of cholesteatoma formation $^{2}$ and was frequently associated with chronic secreatory otitis media ${ }^{3}$. Blockage causing the insufficient ventilation to mastoid and epitympanum could be anywhere in its ventilation pathway and could be independent of eustachian tube function. Marchioni et al in their study introduced with a condition known as selective epitympanic dysventilation syndrome comprising epitympanic dysventilation along with attic retraction and cholesteatoma even in presence of normal eustachian tube function ${ }^{4}$. This is also evident in studies that anterior tympanostomy tube was of no use in treatment of the attic retraction. In tympanoplasty restoration of ventilation is equally important as reconstruction of sound transformer mechanism. During tympanoplasty, effort of clearing the distorted pathway of ventilation to individual compartment would restore the function of that compartment. This would ultimately as a whole restore the functional and anatomical harmony of middle ear cleft postoperatively.

Poor ventilation to epitympanum and mastoid antrum is manifested clinically with the presence of obstructions in the pathway of ventilation such as mucosal adhesions in and around tympanic isthmus, ossio-myringopexy, and epitympanic cholesteatoma. Radiological manifestation of poor ventilation of these compartments shows hypocellular mastoid air cells along with stagnant fluid shadows in mastoid antrum and epitympanum. Exploration of pathway and removal of obstruction is needed to restore ventilation to these compartments. Two approaches are commonly practiced for this purpose. One is cortical mastoidectomy and water test to evaluate epitympanic patency along with removal of blockage. Another one is extended atticotomy, removal of blockage along with reconstruction of attic wall. Present study depicts a novel approach addressing the role of transcanal endoscopic antrostomy hole in improving the poor ventilation to epitympanum and mastoid antrum during tympanoplasty. Study with similar approach was not found in Otolaryngology literatures.

The selected point mentioned in this study for bony dissection for transcanal antrostomy hole to reach mastoid antrum was found safe and effective in every case. About $3 \mathrm{~mm}$ deep transcanal bony dissection purely under endoscopic guidance at this selected point created a tiny passage from external auditory canal to mastoid antrum in all ears with out facing technical complexity and failure. The author named this selected point for antrostomy hole as Shawkat's point according to his own name.
This newly created $2.5 \mathrm{~mm}$ to $3 \mathrm{~mm} \mathrm{~mm}$ ranged wide tiny aperture allowed observing the condition of mucosa at entrance arena of mastoid antrum and also allowed water test for epitympanic patency in all ears. In most of the cases the mucosa was seen healthy. Only in few cases it was slightly inflamed. After removal of obstruction from ventilation pathway, water test is done to ensure the restoration of ventilation from mesotympanum to mastoid antrum through epitympanic corridor. Water test for epitympanic patency involves observation of passage of water from mastoid antrum through epitympanum to mesotympanum. Free passage of water indicates well patency of epitympanum.

The commonest reason of blockage of epitympanic patency in this study was mucosal adhesions around tympanic isthmus along with complete tensor fold. In few cases, severe mucosal adhesion at the level of body of incus required removal of incus to secure epitympanic patency. Tympanoplasty in presence of inflamed middle ear mucosa with dysfunctional eustachian tube put the healing middle ear under threat of insufficient aeration during immediate postoperative period. To overcome such risk, it is customary to use ventilation tube as an additional pathway of aeration to middle ear. Armstrong, Plester, Pulec introduced the intubation through graft, Adrados, Rojo introduced postauricular placement of drainage tube in the mastoid antrum and Portmann introduced the subannular ventilation tube ${ }^{5,6}$. In this study it has been evident that the temporarily placed tube was found performing initially as drainage of secretion and thereafter as additional ventilation pathway to middle ear via mastoid antrum. Rigidity and stability of tiny antrostomy hole provided by its bony base allowed easy removal and reinsertion of stagnant ventilation tubes after cleaning. The judgment for placement of temporary tube according to the load of inflammation of mucosa was found justifiable since both groups showed improved aeration of middle ear cleft in their post operative radiological images. The sealing of this miniature antrostomy hole didn't involve any extra procedure during tympanoplasty. Sealing after removal of ventilation tube involved a very minimal invasive procedure in outpatient department. The postoperative healing of skin at the antrostomy site in all ears was found complete and without any inward growth of skin into mastoid antrum. It was possible probably due to miniature wideness of antrostomy hole.

A hypothetical comparison between those traditional procedures and the present endoscopic surgical procedure in improving postoperative ventilation to epitympanum and mastoid antrum shows that the present endoscopic procedure is less invasive and more mucosal epithelium preservative then those conventional procedures.

Study of Tanabe et al showed that preservation of mastoid mucosa played vital role in recovery of mastoid aeration after surgery ${ }^{7}$. 
In this study the inflamed mucosa of mastoid was also preserved with the hope that this condition of mucosa would be reversed after securing mastoid antrum ventilation. Improved aeration of mastoid antrum in postoperative radiological images produced supportive evidence in favor of this mucosa preservation policy.

In this study it has been observed that the temporarily placed ventilation tube in to antrostomy hole could maintain immediate postoperative ventilation of middle ear successfully. This initial success offers the expectation that this antrostomy hole could also be used for placing ventilation tube for long term aeration of middle ear. Recently Yung described a new method with success engaging placement of a permanent mastoid vent tube externally into mastoid antrum to improve ventilation of atelectatic middle ears ${ }^{8}$. Motivated by the present success and Yung's work, in author's another ongoing unpublished study the author has placed ventilation tube for mid term basis in two conventional ventilation tube failure cases and got promising result. Thus this could be anticipated that in future, this transcanal antrostomy hole will be considered for placing permanent mastoid ventilation tube for treating persistent atelectasis of middle ear.

The present study was a single centered study by a single author. It is the author's expectation and belief that in future, multi-centered study with multiple authorities will definitely upgrade this new technique in regards of improving ventilation to epitympanum and mastoid antrum.

\section{CONCLUSIONS}

This new endoscopic transcanal antrostomy procedure was found safe and effective in securing ventilation to epitympanum and mastoid antrum in retrograde fashion. Bony dissection at the selected point of external auditory canal for this procedure was appeared technically simple and without failure. The tiny antrostomy hole didn't require much effort to manage it and did show complete sealing with healthy skin without any complication. It could be apprehended that in future, this antrostomy passage could be used for placing mastoid ventilation tube for long term basis in treating persistent atelectatic middle ear.

\section{DISCLOSURE}

All the authors declared no competing interest.

\section{References}

1. Takahashi H, Honjo I, Naito Y, Miura M, Tanabe M, Hasebe S. Cause of posterior canal wall retraction after surgery from the viewpoint of mastoid conditions. Am J Otol. 1998;19(2):131-5.

2. Hasebe S, Takahashi H, Honjo I, Miura M, Tanabe M. Mastoid condition and clinical course of cholesteatoma. ORL J Otorhinolaryngol Relat Spec. 2001;63(3):160-4.

3. Sadé J, Fuchs C. Secretory otitis media in adults: II. The role of mastoid pneumatization as a prognostic factor. Ann Otol Rhinol Laryngol. 1997;106(1):37-40.

4. Marchioni D, Alicandri-Ciufelli M, Molteni G, Artioli FL, Genovese E, Presutti L. Selective epitympanic dysventilation syndrome. Laryngoscope. 2010;120(5):1028-33.

5. Hassan M. Mostafa, Salem M. Abdel-Latif and Shaaban B. M. Shaaban. Tympanoplasty with a drain tube in the mastoid antrum. The Journal of laryngology and Otology. 1979; 93: 267-270.

6. Harish S. The Clinical Study of Mastoid Ventilation Tube in Tympanoplasty [dissertation]. Rajiv Gandhi University of Health Sciences, Karnataka. 2006.

7. Tanabe M(1), Takahashi H, Honjo I, Hasebe S, Sudo M. Factors affecting recovery of mastoid aeration after ear surgery. Eur Arch Otorhinolaryngol. 1999;256(5):220-3.

8. Yung M. The Young Percutaneous Mastoid Vent. Arch Otolaryngol Head Neck Surg. 1999;125:964-968. 